Supporting Information for

\title{
Ordered Hierarchical Ag Nanostructures as Surface Enhanced Raman Scattering Platforms for (Bio)chemical Sensing and Pollutants Monitoring
}

Junxiang Xiang 1,a), Yunxia Wang 2,a), Yupeng Wu ${ }^{1}$, Hui Fang ${ }^{1}$, Langquan Shui ${ }^{1}, \mathrm{Ze}$ Liu $1,3, *$ and Tao Ding ${ }^{2, *}$,

${ }^{1}$ Department of Engineering Mechanics, School of Civil Engineering, Wuhan

University, Wuhan, Hubei 430072, China

${ }^{2}$ Key Laboratory of Artificial Micro/Nano Structure of Ministry of Education, School of Physics and Technology, Wuhan University, Wuhan, 430072, China.

${ }^{3}$ State Key Laboratory of Water Resources \& Hydropower Engineering Science,

Wuhan University, Wuhan 430072, China

a) These authors contributed equally.

*Corresponding authors'E-mails: ze.liu@whu.edu.cn (Z.Liu); t.ding@whu.edu.cn (T. Ding). 

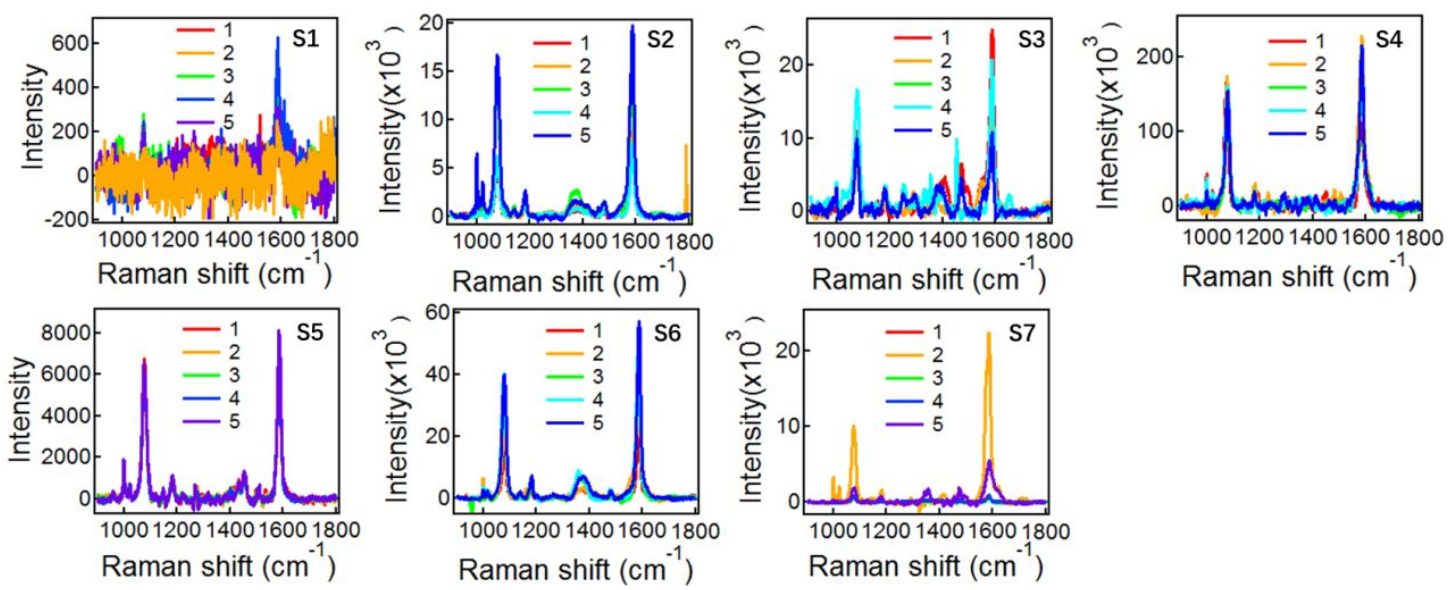

Figure S1. SERS spectra of 4-MBA on S1-S7, which were randomly collected over 5 spots for each sample. 

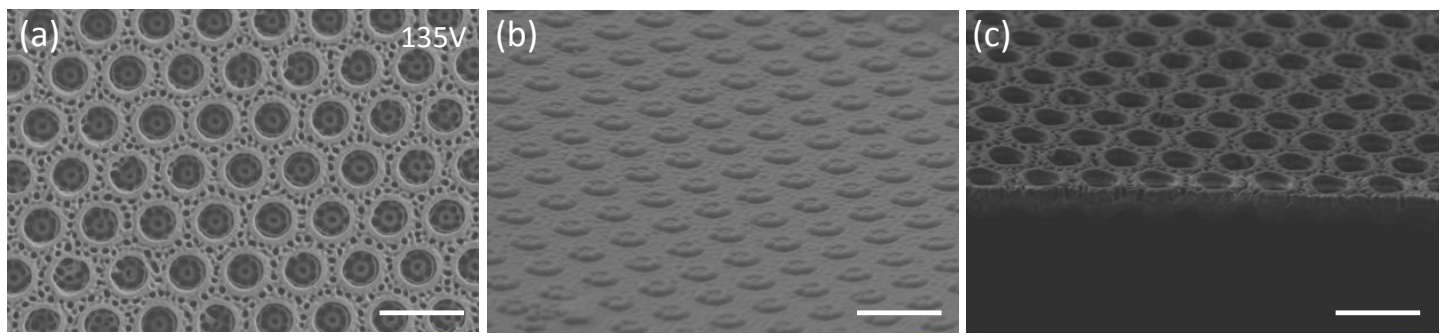

Figure S2 Hierarchical AAO template obtained by oxidation with $0.3 \mathrm{M}$ phosphoric acid solution. The anodizing voltage and time were $135 \mathrm{~V}$ and $2 \mathrm{~min}$, respectively. (a) top view; (b) back view; (C) side view. Scale bars are $2 \mu \mathrm{m}$. 


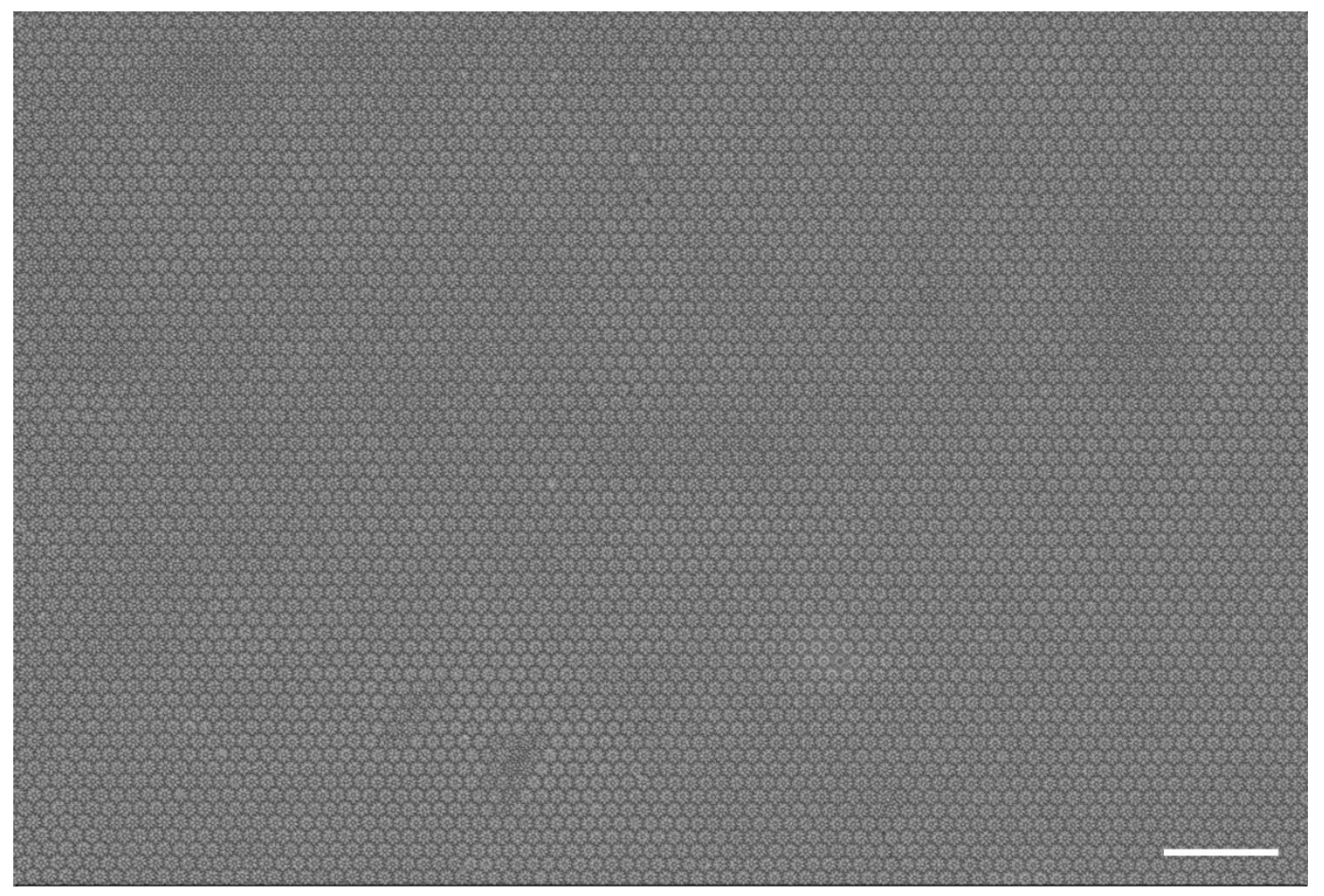

Figure S3 SEM image of large-scale view of molded hierarchical Ag nanostructures by using prepared hierarchical AAO template. Scale bar is $10 \mu \mathrm{m}$ 


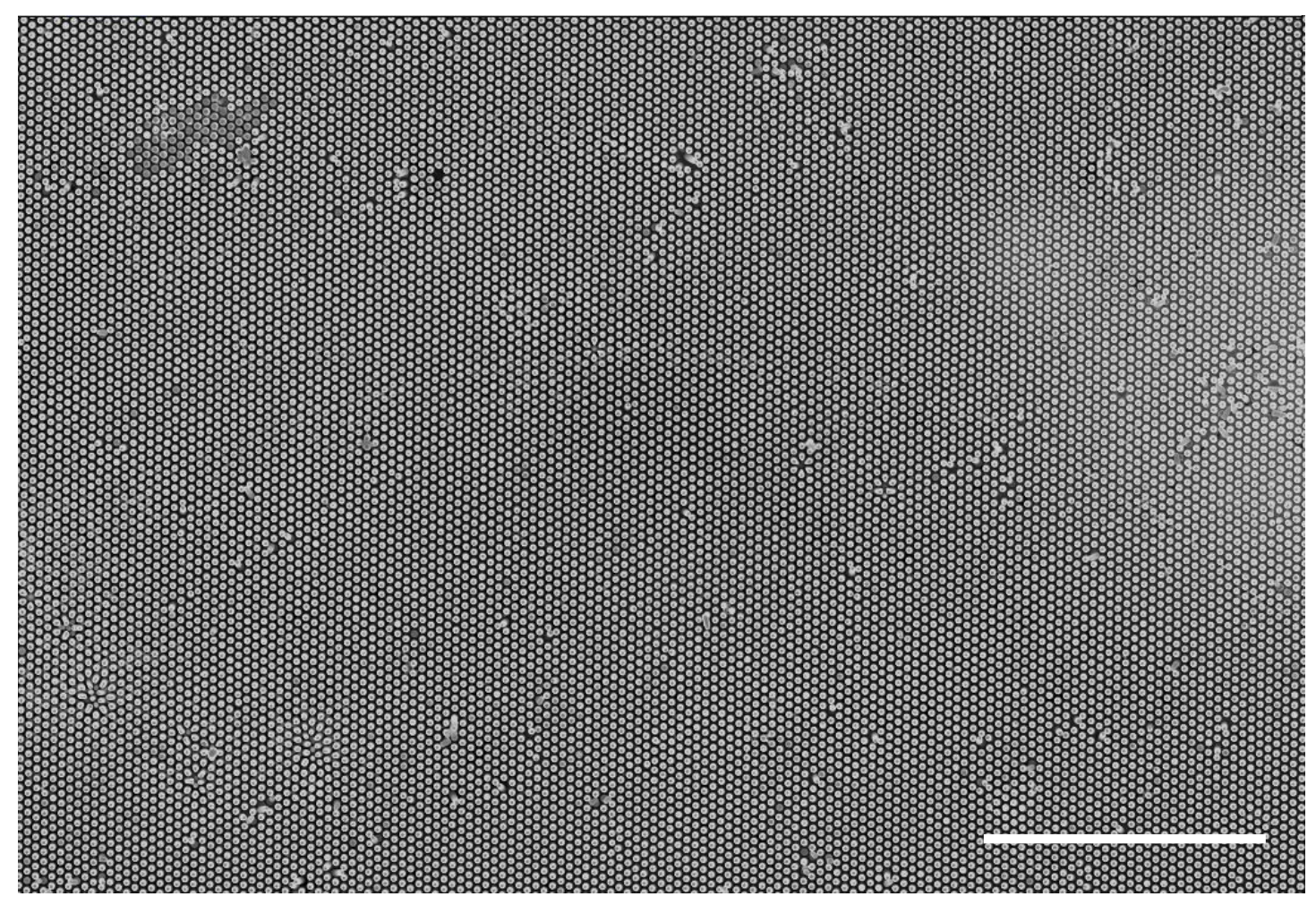

Figure S4 SEM image of molded Ag nanopillars (S7) by using a usual AAO template

(T7). Scale bar is $10 \mu \mathrm{m}$. 


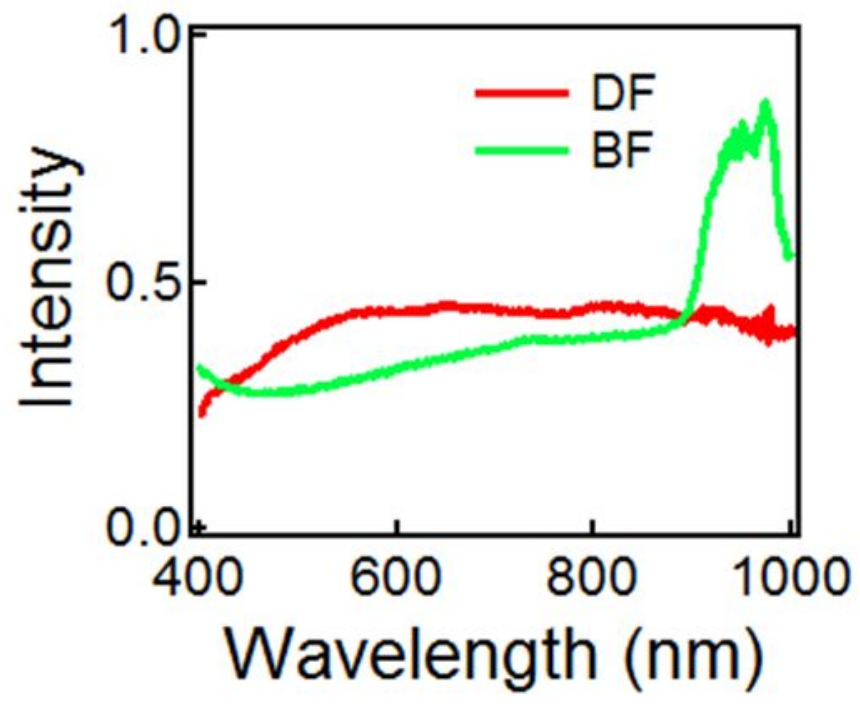

Figure S5 Reflection and scattering spectra of the hierarchical Ag nanostructures (S5). 


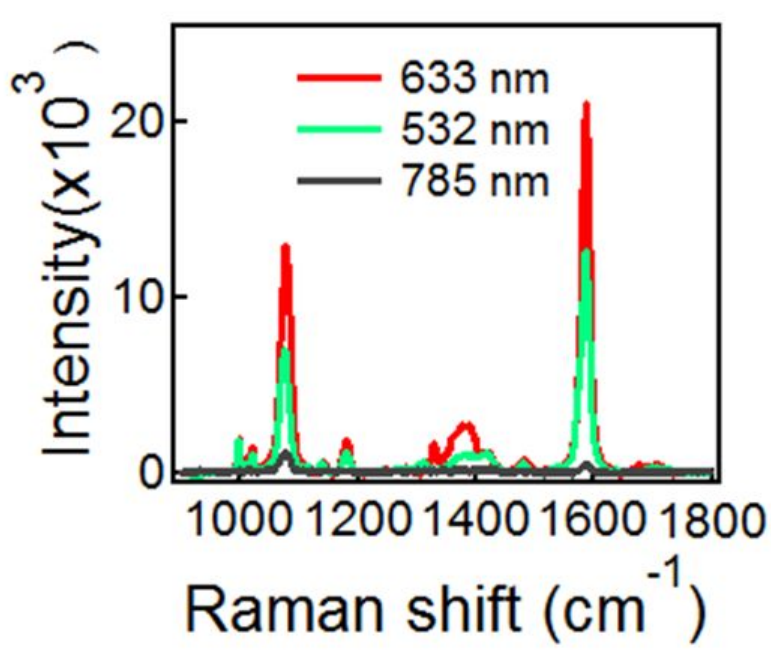

Figure S6 SERS of 4-MBA adsorbed on hierarchical Ag nanostructures (S5) at different excitation wavelengths. 

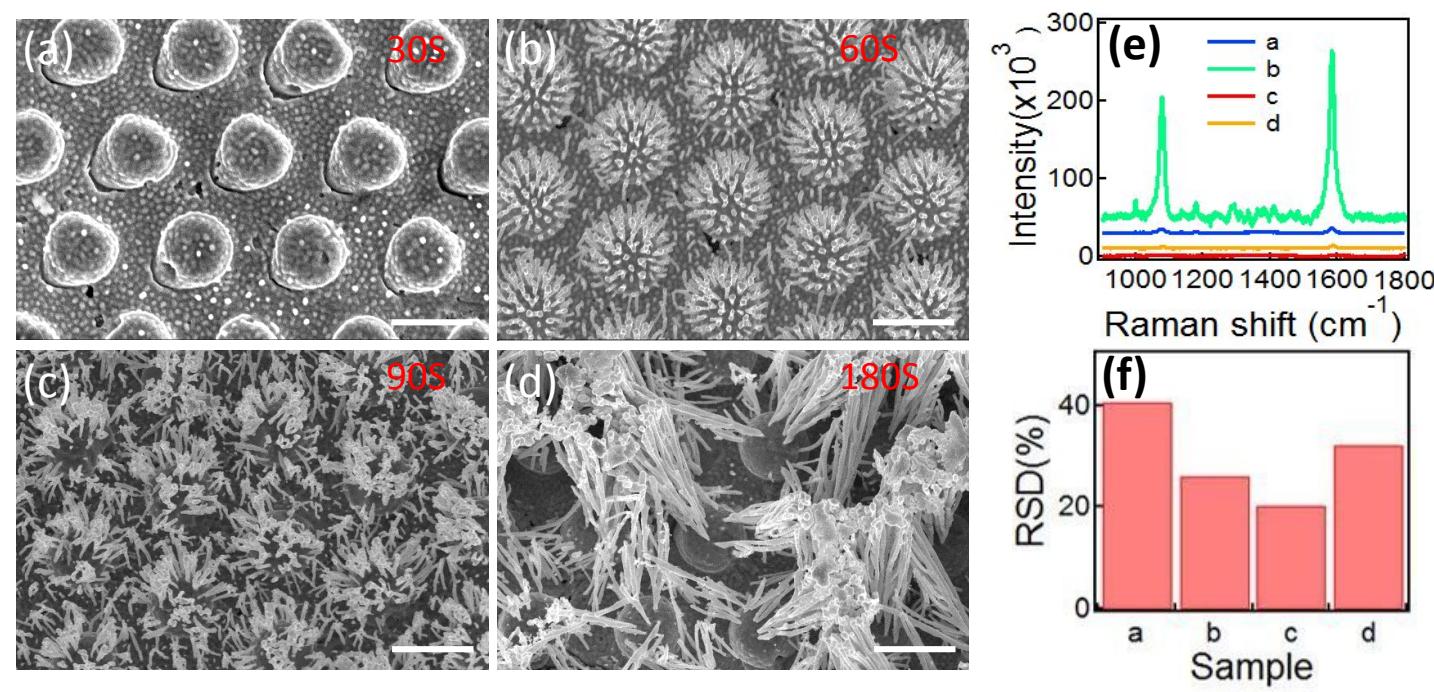

Figure S7 Influence of the height of nanopillars on the SERS performances in Ag hierarchical structures. (a-d) SEM images of Ag hierarchical nanopillars with different heights, which is prepared by nanomolding with T4 molds. The depth of nanoholes in T4 molds were varied by controlling the anodizing reaction time. (e)-(f) SERS spectra from the samples in (a)-(d), and the corresponding RSD values (f). Scale bars are $1 \mu \mathrm{m}$. 